\title{
Refoundation of the Cell Method by means of Augmented Dual Grids
}

\author{
Lorenzo Codecasa ${ }^{1}$ \\ ${ }^{1}$ Dipartimento di Elettronica e Informazione, Politecnico di Milano, Milan, Italy, codecasa@elet.polimi.it
}

\begin{abstract}
The Cell Method (CM) for the discretization of electromagnetic boundary value does not specify how to formulate boundary conditions and how to determine energetic quantities. These drawbacks are here removed reformulating CM by means of a pair of augmented dual grids and by proving a general result for introducing energetic quantities.
\end{abstract}

Index Terms-Computational Electromagnetics, Cell Method, Boundary conditions, Energetic quantities

\section{INTRODUCTION}

As it is well known, in CM the discretization of electromagnetic problems is based on a pair of dual grids, in general polyhedral [8]. Integral electromagnetic quantities are associated to oriented geometric elements of the dual grids in such a way that electromagnetic balance equations are naturally discretized as exact equations. Constitutive relations are discretized by means of approximate equations relating the introduced integral quantities.

The formulation of CM suffers of two serious drawbacks. Firstly, the spacial boundary of a problem is not properly dealt with. In fact the electromagnetic balance equations relative to the geometric elements of the dual grid which intersect the boundary are not in general exact. Besides no general approach for discretizing boundary conditions exists. Secondly, it is not known how to introduce energetic quantities [2].

In this paper all these drawbacks of CM are removed. Firstly, the dual grid is augmented by introducing a boundary dual grid in such a way that all integral electromagnetic quantities can be introduced on the boundary. Secondly a general result is proven for approximating energetic quantities in CM. These two results allow to correct inexact balance equations, lead to the discretization of boundary conditions and allow to introduce energetic quantities. They also lead to exact discrete counterparts of energetic balance equations.

\section{AUgmented DUAL GRIDS}

Let $\mathcal{G}$ be a primal grid discretizing a spacial region $\Omega$, having $n_{n}$ nodes, $n_{e}$ edges, $n_{f}$ faces and $n_{v}$ volumes, and let $\tilde{\mathcal{G}}$ be an oriented dual having $n_{\tilde{n}}=n_{v}$ nodes, $n_{\tilde{e}}=n_{f}$ edges, $n_{\tilde{f}}=n_{e}$ faces and $n_{\tilde{v}}=n_{n}$ volumes. As it is well known [2], the topological description of the primal grid $\mathcal{G}$ is provided by the $n_{e} \times n_{n}$ edge-node incidence matrix $\mathbf{G}$, the $n_{f} \times n_{e}$ face-edge incidence matrix $\mathbf{C}$ and the $n_{v} \times n_{f}$ volumeface incidence matrix $\mathbf{D}$. Similarly the topological description of the dual grid $\tilde{\mathcal{G}}$ is provided by the $n_{\tilde{e}} \times n_{\tilde{n}}$ edge-node incidence matrix $\tilde{\mathbf{G}}=-\mathbf{D}^{T}$, the $n_{\tilde{f}} \times n_{\tilde{e}}$ face-edge incidence matrix $\tilde{\mathbf{C}}=\mathbf{C}^{T}$ and the $n_{\tilde{v}} \times n_{\tilde{f}}$ volume-face incidence matrix $\tilde{\mathbf{D}}=-\mathbf{G}^{T}$.

Let now $\mathcal{G}_{b}$ be the restriction of the primal grid $\mathcal{G}$ to the boundary $\partial \Omega$ of $\Omega$, having $n_{n_{b}}$ nodes, $n_{e_{b}}$ edges and $n_{f_{b}}$ faces. Let $\tilde{\mathcal{G}}_{b}$ be the dual of grid $\mathcal{G}_{b}$ Such dual grid $\tilde{\mathcal{G}}_{b}$ has $n_{\tilde{n}_{b}}=n_{f_{b}}$ nodes obtained by the non-empty intersections with $\partial \Omega$ of the edges of $\tilde{\mathcal{G}}$, has $n_{\tilde{e}_{b}}=n_{e_{b}}$ edges obtained by the non-empty intersection with $\partial \Omega$ of the faces of $\tilde{\mathcal{G}}$ and has $n_{\tilde{f}_{b}}=n_{n_{b}}$ faces obtained by the non-empty intersection with $\partial \Omega$ of the volumes of $\tilde{\mathcal{G}}$. Let $\tilde{\mathbf{D}}_{b}$ be the $n_{\tilde{v}} \times n_{\tilde{f}_{b}}$ incidence matrix of the $n_{\tilde{v}}$ volumes of $\tilde{\mathcal{G}}$ with the $n_{\tilde{f}}$ faces of $\tilde{\mathcal{G}}_{b}$. Similarly let $\tilde{\mathbf{C}}_{b}$ be the $n_{\tilde{f}} \times n_{\tilde{e}_{b}}$ incidence matrix of the $n_{\tilde{f}}$ faces of $\tilde{\mathcal{G}}$ with the $n_{\tilde{e}}$ edges of $\tilde{\mathcal{G}}_{b}$ and let $\tilde{\mathbf{G}}_{b}$ be the $n_{\tilde{e}} \times n_{\tilde{n}_{b}}$ incidence matrix of the $n_{\tilde{e}}$ edges of $\tilde{\mathcal{G}}$ with the $n_{\tilde{n}}$ nodes of $\tilde{\mathcal{G}}_{b}$. The union of the dual grids $\tilde{\mathcal{G}}$ and $\tilde{\mathcal{G}}_{b}$ define an augmented oriented dual grid $\tilde{\mathcal{G}}_{a}$. The volume-face, face-edge and edge-node incidence matrices of $\tilde{\mathcal{G}}_{a}$, named $\tilde{\mathbf{G}}_{a}, \tilde{\mathbf{C}}_{a}$ and $\tilde{\mathbf{D}}_{a}$, can be derived from previous incidence matrices as follows

\section{Property 1:}

$$
\begin{aligned}
\tilde{\mathbf{G}}_{a} & =\left[\begin{array}{cc}
\tilde{\mathbf{G}} & \tilde{\mathbf{G}}_{b} \\
\mathbf{0} & -\tilde{\mathbf{C}}_{b}^{T} \tilde{\mathbf{C}} \tilde{\mathbf{G}}_{b}
\end{array}\right], \\
\tilde{\mathbf{C}}_{a} & =\left[\begin{array}{cc}
\tilde{\mathbf{C}} & \tilde{\mathbf{C}}_{b} \\
\mathbf{0} & -\tilde{\mathbf{D}}_{b}^{T} \tilde{\mathbf{D}} \tilde{\mathbf{C}}_{b}
\end{array}\right], \\
\tilde{\mathbf{D}}_{a} & =\left[\begin{array}{ll}
\tilde{\mathbf{D}} & \tilde{\mathbf{D}}_{b}
\end{array}\right] .
\end{aligned}
$$

\section{Discrete EnERgetic QUANTITIES}

Let $\boldsymbol{x}$ be an array of circulations along the edges of $\mathcal{G}$, or an array of fluxes through the faces of $\mathcal{G}$, of a vector field $\mathbf{X}(\mathbf{r})$, being $\mathbf{r}$ the position vector, and let $\tilde{\boldsymbol{y}}$ be an array of fluxes through the faces of $\tilde{\mathcal{G}}$, or an array of circulations along the edges of $\tilde{\mathcal{G}}$, of a vector field $\mathbf{Y}(\mathbf{r})$. It results in

Property 2: For any pair of oriented dual grids $\mathcal{G}, \tilde{\mathcal{G}}$, not necessarily polyhedral, the discrete energetic quantity

$$
\boldsymbol{x}^{T} \tilde{\boldsymbol{y}}
$$

provides an approximation of the energetic quantity

$$
\int_{\Omega} \mathbf{X}(\mathbf{r}) \cdot \mathbf{Y}(\mathbf{r}) d \Omega .
$$

The convergence of this approximation is at least of first order with respect to the maximum diameter $h_{M}$ of $\mathcal{G}$.

Such result extends a similar result in the case in which $x$ is an array of values at the nodes of $\mathcal{G}$, or an array of integrals over the volumes of $\mathcal{G}$, of a scalar field $x(\mathbf{r})$ and $\tilde{\boldsymbol{y}}$ is an array 
of integrals over the volumes of $\tilde{\mathcal{G}}$, or an array of values at the nodes of $\tilde{\mathcal{G}}$, of a scalar field $y(\mathbf{r})$. Thus in all cases (1) provides an approximation of an energetic quantity.

Analogous results are derived for the discrete energetic quantity

$$
\boldsymbol{x}_{b}^{T} \tilde{\boldsymbol{y}}_{b}
$$

in which $\tilde{\boldsymbol{y}}_{b}$ is an array of quantities associated to the faces, nodes or edges of the boundary dual grid $\tilde{\mathcal{G}}_{b}$ and $\boldsymbol{x}_{b}$ is an array of quantities associated to the nodes, edges or faces of the boundary primal grid $\mathcal{G}_{b}$, which can be selected from the array $\boldsymbol{x}$ of quantities associated to the nodes, edges or faces of the primal grid $\mathcal{G}$ respectively by $\boldsymbol{x}_{b}=\tilde{\mathbf{G}}_{b}^{T} \boldsymbol{x}, \boldsymbol{x}_{b}=\tilde{\mathbf{C}}_{b}^{T} \boldsymbol{x}$ or $\boldsymbol{x}_{b}=\tilde{\mathbf{D}}_{b}^{T} \boldsymbol{x}$.

In particular in case $\boldsymbol{x}_{b}$ is the array of the circulations along the edges of $\mathcal{G}_{b}$ of a vector field $\mathbf{X}(\mathbf{r})$ and $\tilde{\boldsymbol{y}}_{b}$ is the array of the circulations along the edges of $\tilde{\mathcal{G}}_{b}$ of a vector field $\mathbf{Y}(\mathbf{r})$, (2) provides an approximation of

$$
\int_{\partial \Omega} \mathbf{X}(\mathbf{r}) \times \mathbf{Y}(\mathbf{r}) \cdot \mathbf{n}(\mathbf{r}) d \Omega
$$

in which $\mathbf{n}(\mathbf{r})$ is the outward unit vector normal to $\partial \Omega$ at $\mathbf{r}$.

\section{CM DISCRETIZATION OVER AUGMENTED DUAL GRIDS}

For the sake of simplicity hereafter an electromagnetic boundary value problem in the frequency domain is considered. As usual the array $e$ of the circulations of the electric field $\mathbf{E}(\mathbf{r})$ along the edges of $\mathcal{G}$ and the array $\boldsymbol{b}$ of the fluxed of the magnetic induction $\mathbf{B}(\mathbf{r})$ through the faces of $\mathcal{G}$ are introduced. Besides the array $\tilde{h}$ of the circulations of the magnetic field $\mathbf{H}(\mathbf{r})$ along the edges of $\tilde{\mathcal{G}}$ and the arrays $\tilde{\boldsymbol{d}}$, $\tilde{j}$ of the fluxes of the electric displacement $\mathbf{D}(\mathbf{r})$ and of the electric current $\mathbf{J}(\mathbf{r})$ through the faces of $\tilde{\mathcal{G}}$ are introduced. In addition, the array $\tilde{\boldsymbol{h}}_{b}$ with the circulations of $\mathbf{H}(\mathbf{r})$ along the edges of $\tilde{\mathcal{G}}_{b}$ and the array arrays $\tilde{\boldsymbol{d}}_{b}, \tilde{\boldsymbol{j}}_{b}$ of the fluxes of $\mathbf{D}(\mathbf{r})$ and $\mathbf{J}(\mathbf{r})$ through the faces of $\tilde{\mathcal{G}}_{b}$ are introduced. In this way the following arrays of quantities associated to the augmented dual grid $\tilde{\mathcal{G}}_{a}$ are introduced

$$
\tilde{\boldsymbol{h}}_{a}=\left[\begin{array}{c}
\tilde{\boldsymbol{h}} \\
\tilde{\boldsymbol{h}}_{b}
\end{array}\right], \quad \tilde{\boldsymbol{d}}_{a}=\left[\begin{array}{c}
\tilde{\boldsymbol{d}} \\
\tilde{\boldsymbol{d}}_{b}
\end{array}\right], \quad \tilde{\boldsymbol{j}}_{a}=\left[\begin{array}{c}
\tilde{\boldsymbol{j}} \\
\tilde{\boldsymbol{j}}_{b}
\end{array}\right] .
$$

These quantities allow to write exact balance equations both on grid $\mathcal{G}$ and $\tilde{\mathcal{G}}_{a}$. Balance equations over the primal grid $\mathcal{G}$ are written as usual. For instance Faraday's equation is

$$
\mathbf{C e}=-i \omega \boldsymbol{b},
$$

in which $\omega$ is the angular frequency. Balance equations over the augmented dual grid $\tilde{\mathcal{G}}_{a}$ are written in a similar way. For instance Ampère-Maxwell equations is

$$
\tilde{\mathbf{C}}_{\mathrm{a}} \boldsymbol{h}_{a}=i \omega \boldsymbol{d}_{a}+\boldsymbol{j}_{a},
$$

In particular, from such equation, recalling property 1 , it follows

$$
\tilde{\mathbf{C}} \boldsymbol{h}+\tilde{\mathbf{C}}_{b} \boldsymbol{h}_{b}=i \omega \boldsymbol{d}+\boldsymbol{j},
$$

which corrects the inexact balance equations on $\tilde{\mathcal{G}}$ in $\mathrm{CM}$ [2].
Constitutive equations are discretized as usual by means of the quantities over $\mathcal{G}, \tilde{\mathcal{G}}$. For instance electric and magnetic constitutive relations take the form [8], [3]

$$
\tilde{\boldsymbol{d}}=\mathbf{M}_{\varepsilon} \boldsymbol{e}, \quad \tilde{\boldsymbol{h}}=\mathbf{M}_{\nu} \boldsymbol{b} .
$$

Quantity $\boldsymbol{e}_{b}=\tilde{\mathbf{C}}_{b}^{T} \boldsymbol{e}$ over $\mathcal{G}_{b}$ and quantity $\tilde{\boldsymbol{h}}_{b}$ over $\tilde{\mathcal{G}}_{b}$ provide the way for discretizing boundary equations. For instance, admittance boundary conditions over $\partial \Omega$

$$
(\mathbf{H}(\mathbf{r}) \times \mathbf{n}(\mathbf{r})) \times \mathbf{n}(\mathbf{r})=Y(\mathbf{r}) \mathbf{E}(\mathbf{r}) \times \mathbf{n}(\mathbf{r})
$$

are discretized by approximate equations of the form [4]

$$
\tilde{\boldsymbol{h}}_{b}=\mathbf{M}_{Y} \boldsymbol{e}_{b} .
$$

Energetic quantities can also be determined. For instance the electric energy, magnetic energy, electric power over $\Omega$ and flux of Poynting's vector across $\partial \Omega$ are discretized respectively by

$$
\frac{1}{4} \tilde{\boldsymbol{d}}^{*} \boldsymbol{e}, \quad \frac{1}{4} \tilde{\boldsymbol{h}}^{*} \boldsymbol{b}, \quad \frac{1}{2} \tilde{\boldsymbol{j}}^{*} \boldsymbol{e}, \quad \frac{1}{2} \tilde{\boldsymbol{h}}_{b}^{*} \boldsymbol{e}_{b}
$$

in which $*$ indicates complex conjugate transpose.

By multiplying (3) on the left by $\tilde{\boldsymbol{h}}^{*}$, multiplying the complex conjugate transpose of (3) on the right by $\tilde{e}$ and subtracting, the discrete counterpart of Poynting's theorem follow

$$
\frac{1}{2} \tilde{\boldsymbol{h}}_{b}^{*} \boldsymbol{e}_{b}=2 i \omega\left(\frac{1}{4} \tilde{\boldsymbol{h}}^{*} \boldsymbol{b}-\frac{1}{4} \tilde{\boldsymbol{d}}^{*} \boldsymbol{e}\right)+\frac{1}{2} \tilde{\boldsymbol{j}}^{*} \boldsymbol{e}
$$

Thus exact balance equations are disclosed for the introduced discrete energetic quantities.

By substituting the discrete electric constitutive equation into the expression of the discrete electric energy, it follows that matrix $\mathbf{M}_{\varepsilon}$ not only allows to approximate $\tilde{d}$ given $e$ but also allows to approximate the electric energy given $e$. This double purpose of $\mathbf{M}_{\varepsilon}$ can be revealed in the same way for all matrices in constitutive relations and boundary conditions.

\section{Numerical RESUlts}

A $T M_{10}$ is injected into a section of rectangular waveguide terminated by a matching admittance boundary condition. This problem is discretized over a tetrahedral grid with 32,141 nodes and an augmented dual grid [4]. The flux of Poynting's vector at the port is estimated with $1 \%$ accuracy by (2).

\section{Conclusions}

The full paper will include more in-depth description, complete proofs and details of the numerical analysis.

\section{REFERENCES}

[1] L. Codecasa and F. Trevisan, "Constitutive equations for discrete electromagnetic problems over polyhedral grids,"Journal of Computational Physics, Vol. 225 No. 2, pp. 1894-1918, 2007.

[2] E. Tonti, "Finite formulation of electromagnetic field," IEEE Transactions on Magnetics, Vol. 38, No. 2, pp. 333-336 , 2002.

[3] L. Codecasa and M. Politi, "Use of barycentric dual grids for the solution of frequency domain problems by FIT," IEEE Transactions on Magnetics, Vol. 40, No. 2, pp. 1414-1419, 2004.

[4] L. Codecasa, R. Specogna, F. Trevisan, "Discrete Geometric Formulation of Admittance Boundary Conditions for Frequency Domain Problems Over Tetrahedral Dual Grids," IEEE Transactions on Antennas and Propagation, Vol. 60, No. 8, pp. 3998-4002, 2012. 